Supporting Information for

\title{
BioSTEAM-LCA: An Integrated Modeling Framework for Agile Life Cycle Assessment of Biorefineries Under Uncertainty
}

\author{
Rui Shi ${ }^{1}$, Jeremy S. Guest ${ }^{1,2}$ \\ ${ }^{1}$ Center for Advanced Bioenergy and Bioproducts Innovation (CABBI), University of Illinois at \\ Urbana-Champaign, Urbana, IL 61801, United States \\ ${ }^{2}$ Department of Civil \& Environmental Engineering, University of Illinois at Urbana-Champaign, \\ Urbana, IL 61801, United States
}

Number of Pages: 10

Number of Figures: 5

Number of Tables: 6 


\section{Table of Contents}

Section S1. Definition of technical terms for agile LCA-TEA.

Section S2. Case study design flows.

Section S3. Key assumptions of sugarcane biorefinery in the case study.

Section S4. Default assumptions on distributions follow the ecoinvent pedigree approach

Section S5. Calculation of energy allocation factor for ethanol and co-products.

\section{Supplementary Figures:}

Figure S1. Detailed design flow for fermentation unit for agile LCA simulation.

Figure S2. Flowsheet depicting main areas of the sugarcane biorefinery

(Excel spreadsheet).

Figure S3. Distribution of energy allocation factor (EAF). $\quad$ S7

Figure S4. Top 10 technology parameters for sugarcane biorefinery that are S8 sensitive to MESP and GHG.

Figure S5. Simplified unified modeling Language class diagram for the core

\section{Supplementary Tables}

Table S1. Definition and exploration of key terms used in the agile LCA methodology following a QSD framework.

Table S2. Detailed composition of sugarcane used in the case study.

Table S3. Stream tables for sugarcane biorefinery (Excel spreadsheet).

Table S4. Monte Carlo simulation results for estimating the uncertainties of a gate-to-gate LCA of a sugarcane biorefinery (Excel spreadsheet).

Table S5 Pedigree matrix default probability distributions used in ecoinvent.

Table S6 Empirically based uncertainty factors for the pedigree matrix, these factors are contributing to the square of the geometric standard deviation. 


\section{Section S1. Definition of technical terms for agile LCA-TEA}

Table S1. Definition and exploration of key terms used in the agile LCA methodology following a QSD framework.

\begin{tabular}{ll} 
Terms & Description \\
decision variables & $\begin{array}{l}\text { independent parameters and quantities; variables a process } \\
\text { designer or operator can specify or control. For example: feed flow } \\
\text { rates, operating pressures, retention times, etc. }\end{array}$ \\
\hline technology parameters & $\begin{array}{l}\text { parameters that are intrinsic to the materials and design of a system; } \\
\text { used in design and simulation to predict technical performance. For } \\
\text { example: maximum specific growth rate of fermenters, aeration } \\
\text { efficiency, etc. }\end{array}$ \\
\hline contextual parameters & $\begin{array}{l}\text { non-technological factors that influence metrics. For example: } \\
\text { factors that vary depending on the location, policies, end users, time } \\
\text { of year, policy incentives, carbon intensity of agricultural industry, } \\
\text { etc. }\end{array}$ \\
\hline decision space & all possible combinations of decision variables that can be specified. \\
\hline simulation space & $\begin{array}{l}\text { all possible combinations of decision variables, technology } \\
\text { parameters, and contextual parameters that can exist. }\end{array}$ \\
\hline design algorithms & $\begin{array}{l}\text { detailed design rules established to automate the detailed design of } \\
\text { a system based on decision variables, technology parameters, and } \\
\text { contextual parameters. For example: algorithms for the surface area } \\
\text { of a shell and tube heat exchanger based on log mean temperature } \\
\text { difference, heat transfer coefficient, flow rate, heat capacity, and } \\
\text { temperature change or outlet temperature. }\end{array}$ \\
\hline detailed inventory data as a function of decision variables, \\
technology parameters, and contextual parameters. For example: \\
material usage, power, steam, cooling water, chilled water, etc. \\
\hline mantentory data
\end{tabular}

\section{Section S2. Case study design flows}

In this agile life cycle assessment module, we automated the process of generating inventory inputs of each unit operation by automating the design and simulation of the biorefinery as a function of detailed design decisions, assumptions about technologies and unit operations, and contextual parameters. Below, Figure S1 shows the design flow of a fermenter as an example. 


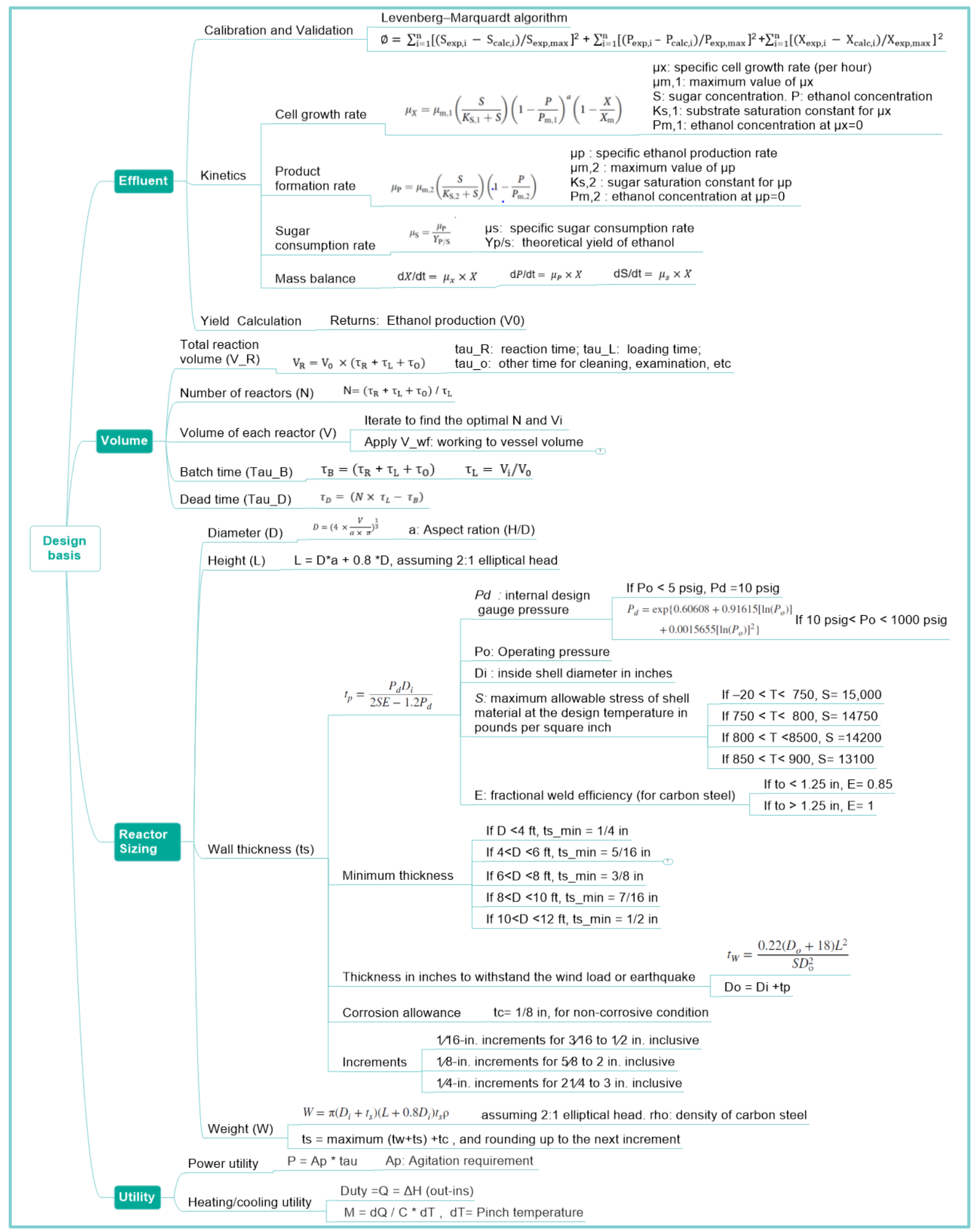

Figure S1. Detailed design flow for fermentation unit. 


\section{Section S3. Key assumptions of sugarcane biorefinery in the case study}

Capacity of biorefinery and composition of sugarcane in this exemplary study were selected to be 8000 metric ton stems per day with sugar content of $49.7 \%$ (dry matter basis) based on a previous publication ${ }^{1}$. Operating days were assumed to be 200 days in a year. Detailed composition of sugarcane can be find below, using data adapted from (Kumar 2018) paper ${ }^{1}$. Flowsheet depicting main areas of the sugarcane biorefinery can be found in Excel spreadsheet.

Table S2. Detailed composition of sugarcane (flowrate, kg per hour).

\begin{tabular}{llllllll}
\hline Ash & Cellulose & Hemicellulose & Lignin & Glucose & Sucrose & Solids & Water \\
\hline 2000 & 20384 & 12027 & 10922 & 4027 & 45640 & 5000 & 233334 \\
\hline
\end{tabular}

Figure S2. Flowsheet depicting main areas of the sugarcane biorefinery (Excel spreadsheet).

(Figure S2 is provided in a Microsoft Excel spreadsheet:

Shi_Guest_Supplementary_Tables_S3_S4_Figure_S2.xlsx)

Table S3. Stream tables and design specifications for sugarcane biorefinery (Excel spreadsheet).

(Table S3 is provided in a Microsoft Excel spreadsheet:

Shi_Guest_Supplementary_Tables_S3_S4_Figure_S2.xlsx)

Table S4. Monte Carlo simulation results for estimating the uncertainties of a gate-to-gate LCA of a sugarcane biorefinery (Excel spreadsheet).

(Table S4 is provided in a Microsoft Excel spreadsheet:

Shi_Guest_Supplementary_Tables_S3_S4_Figure_S2.xlsx) 


\section{Section S4. Default assumptions on distributions follow the ecoinvent pedigree approach}

BioSTEAM-LCA has default probability density functions for uncertain parameters, and also offers users the flexibility of defining their own distributions. For unit impacts, we estimate the uncertainty of all inputs and outputs of each process by applying empirically based uncertainty factors using the pedigree matrix approach (default assumptions on distribution types are shown in Table S3). This approach obtains both base uncertainty (the epistemic error applied to intermediate and elementary exchanges) and pedigree factors following ecoinvent's methodology.

Table S5. Pedigree matrix default probability distributions used in ecoinvent.

\begin{tabular}{|l|l|l|}
\hline Exchange type & Probability distribution type & $\begin{array}{l}\text { Number of } \\
\text { exchanges }\end{array}$ \\
\hline Elementary & Log-normal & 188769 \\
\hline Elementary & Undefined/unknown & 181585 \\
\hline Elementary & Normal & 60 \\
\hline Elementary & Triangular & 34 \\
\hline Intermediate & Log-normal & 133030 \\
\hline Intermediate & Undefined/unknown & 41159 \\
\hline Intermediate & Triangular & 97 \\
\hline Intermediate & Normal & 1 \\
\hline
\end{tabular}

Table S6. Empirically based uncertainty factors for the pedigree matrix, these factors are contributing to the square of the geometric standard deviation.

\begin{tabular}{|l|l|l|l|l|l|}
\hline Indicator score & 1 & 1.05 & 1.1 & 1.20 & 1.50 \\
\hline Reliability & 1.0 & 1.02 & 1.05 & 1.10 & 1.20 \\
\hline Completeness & 1.0 & 1.03 & 1.0 & 1.20 & 1.50 \\
\hline Temporal correlation & 1.0 & 1.01 & 1.02 & & 1.1 \\
\hline Geographical correlation & 1.0 & & 1.20 & 1.50 & 2.0 \\
\hline Further technological correlation & 1.0 & 1.02 & 1.05 & 1.10 & 1.20 \\
\hline Sample size & 1.0 & & & & \\
\hline
\end{tabular}




\section{Section S5. Calculation of energy allocation factor for ethanol and co-products}

The co-product of sugarcane derived ethanol is the excess electricity generated onsite by burning sugarcane bagasse. Energy allocation factor (EAF) between the fuel and the co-produced electricity at biorefinery were quantified under uncertainty using the below equations, where $E_{\text {toh }}$

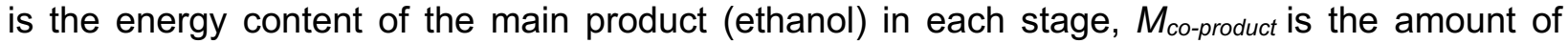
coproduces produced along with main product (excess electricity), and $E_{c o-p r o d u c t}$ is the energy content of the co-products.

$E A F_{\text {at refinery }}=\frac{E_{\text {etoh }}}{\left(E_{\text {etoh }}+M_{c o-\text { product }} \times E_{\text {co-product }}\right)}$

This module uses an agile approach to estimate the EAF. The EAF distribution based on ethanol production and electricity generation under uncertainty are provided in Figure S3. In the GREET Model, the static EAF for sugarcane ethanol is $86 \%{ }^{2}$.

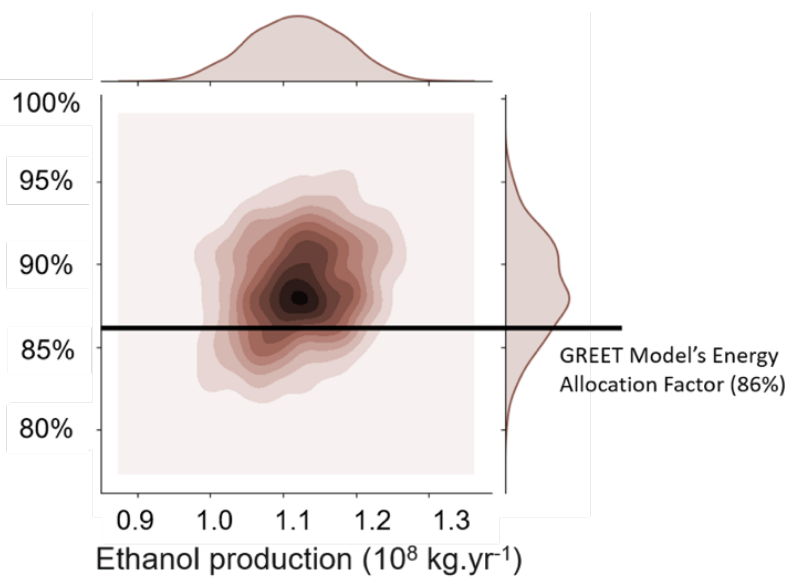

Figure S3. Distribution of energy allocation factor $(E A F)$. Black line indicators the EAF in GREET Model. 


\section{Section S6. Sensitivity analysis results}

This agile LCA approach enables the understanding of the importance of all uncertainty input parameters through Spearman's rank-order correlation using the Monte Carlo results. All process parameters have a triangular uncertainty distribution, more in-depth studies of the limits and uncertainties of input parameters are needed in future work.

\section{(A). Spearman's correlation with MESP}

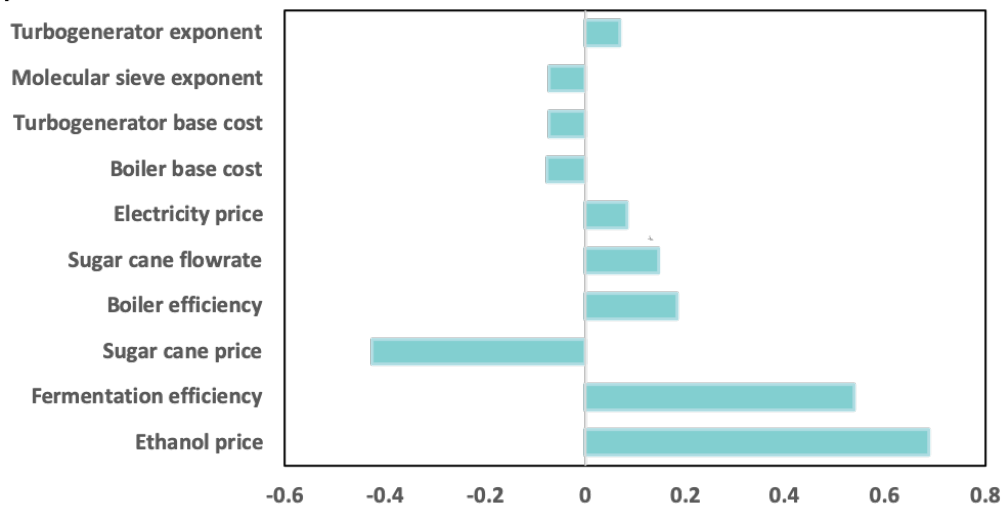

(B). Spearman's correlation with GHG

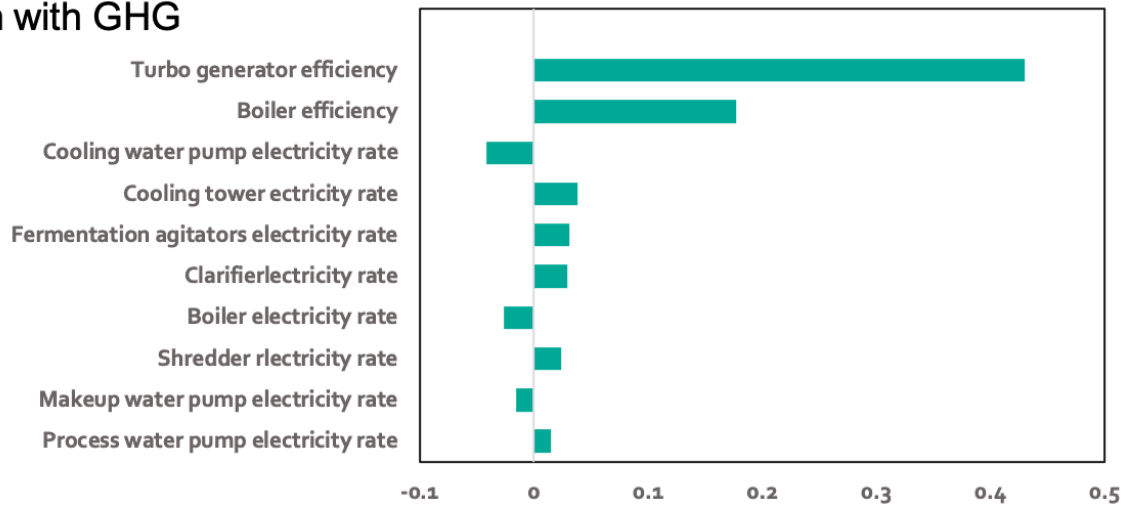

Figure S4. Top 10 technology parameters for sugarcane biorefinery that are $(A)$ sensitive to minimum ethanol selling price (MESP); (B)sensitive to GHG. All process parameters have a triangular uncertainty distribution. 
Section S7. Core classes in BioSTEAM-LCA.

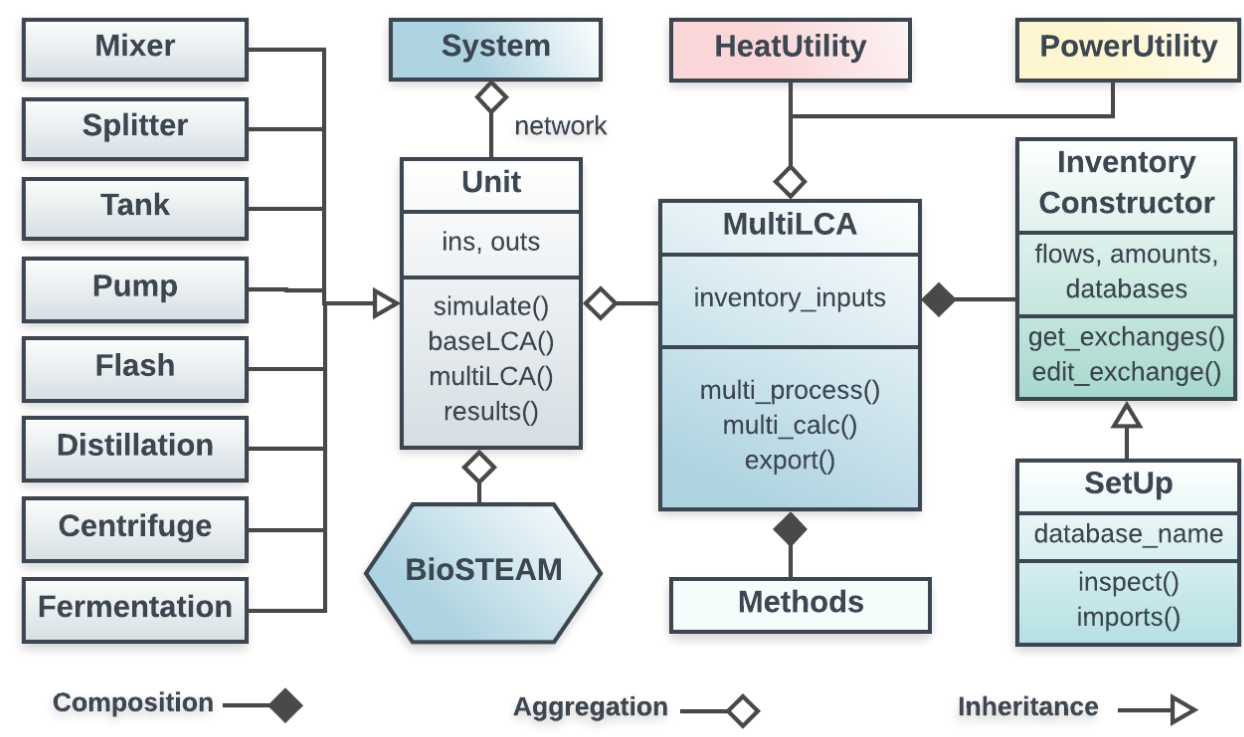

Figure S5. A simplified Unified Modeling Language class diagram depicting the core classes of BioSTEAMLCA. The classes are represented by boxes containing the main attributes (i.e., data fields) and methods (i.e., functional algorithms). The inheritance relationship shows that all unit operations inherit the same layout as the Unit class. The InventoryConstructor inherits attributes (activities and exchanges) from the SetUp class. In aggregation relationships, the labels represent the attributes where the objects are stored. To execute MultiLCA calculation, users must use InventoryConstructor object to define the input and output flows that user selected for each unit process inputs. 


\section{References}

(1) Kumar, D.; Long, S. P.; Singh, V. Biorefinery for Combined Production of Jet Fuel and Ethanol from Lipid-Producing Sugarcane: A Techno-Economic Evaluation. GCB Bioenergy 2018, 10 (2), 92-107. https://doi.org/10.1111/gcbb.12478.

(2) Argonne National Laboratory. The Greenhouse gases, Regulated Emissions, and Energy use in Transportation (GREET) Model. Retrieved from https://greet.es.anl.gov/ (accessed Nov 18, 2019).

(3) Wernet, G.; Bauer, C.; Steubing, B.; Reinhard, J.; Moreno-Ruiz, E.; Weidema, B. The Ecoinvent Database Version 3 (Part I): Overview and Methodology. Int. J. Life Cycle Assess. 2016, 21 (9), 1218-1230. DOI 10.1007/s11367-016-1087-8. 